\title{
A PRÁTICA DE ATIVIDADE FÍSICA EM ADOLESCENTES OBESOS
}

\section{The practice of phisical activity among obese adolescents}

\author{
Cláudia Terra do Nascimento \& Léris Salete Bonfante Haeffner ${ }^{\circ}$
}

\begin{abstract}
Resumo
Diante do nivel altíssimo de sedentarismo entre os jovens em geral e em especial entre adolescentes obesos, realizou-se este estudo, com o objetivo de verificar o nivel de atividade física dos 52 adolescentes obesos, de ambos os sexos ( 27 meninas e 25 meninos), de 10 a 18 anos, em tratamento no Ambulatório de Obesidade na Infância e Adolescência (HUSM), da UFSM. Para este fim, utilizou-se como instrumento de pesquisa uma adaptação do Questionário Internacional de Nivel de Atividade Física (IPAQ - Versão 6). Sua análise foi realizada a partir do critério de Atividade Física Total (AFT), segundo a recomendaçāo atual em saúde, sendo considerados ativos aqueles que obtiveram a somatória de pelo menos 150 minutos por semana de atividade física moderada ou de pelo menos 60 minutos por semana de atividade vigorosa. Os resultados mostraram que, se por um lado o tempo de inatividade foi bastante grande, verificado neste estudo a partir do tempo de assistência à televisāo (em média, quase 5 horas diárias), o nivel de prática de atividade fisica foi muito mais de sedentarismo do que de atividade $(63,46 \%$ de sedentários e $36,54 \%$ de ativos), fato que vem ao encontro da literatura, tanto em relaçăo ao estilo de vida inativo, o qual parece estar cada vez maior na adolescéncia, quanto ao indício do sedentarismo ser maior entre os adolescentes obesos do que em seus pares nāo obesos. Ambas as situaçōes podem explicar os altos percentuais de inatividade encontrados neste estudo entre os adolescentes obesos investigados, denotando a preocupaçăo mundial com o estilo de vida, enquanto um dos grandes responsáveis pelo quadro da epidemia de obesidade na atualidade.
\end{abstract}

Palavras-Chave: Obesidade, Adolescência, Prática de atividade física.

Abstract

Due to the high level of sedentary habits among the young population in general, and specially among obese adolescents, this study was performed. with the objective of verifying the level of physical activity of the 52 obese adolescents, boys and girls ( 27 girls and 25 boys), who have been treated at the Obesity in Childhood and Adolescence Ambulatory (HUSM) trom UFSM. For this purpose, an adaptation of the International Physical Activity Questionnaire (IPAQ - version 6) was used as the investigational instrument. Its analysis was performed from the criteria of Total Physical Activity (AFT), following the current health recommendation, in which, were considered active those who had the score of: At least 150 minutes of moderated physical activity weekly or at least 60 minutes of intense physical activity weekly. The results showed that, not only the time of inactivity was quite long, verified, in this study, by the time spent watching television (an average of 5 hours a day), but also, when it comes to the level of practice of physical activity, the sedentary habits are superior to activity $(63,46 \%$ sedentary to $36.54 \%$ actives), a fact that is in accordance with the literature, not only regarding inactive lifestyle. that seems to be getting more prevalent in the adolescence. but also concerning the evidence that sedentary life style is more pronounced among the obese adolescents than among their non-obese equivalent. Both situations can explain the high rates of inactivity found in this study among the investigated obese adolescents, indicating the world's concern about the lifestyle as one of the major responsible for the present obesity epidemics.

Key Words: Obesity, Adolescence, Practice of physical activity.

\footnotetext{
Autora, Aluna do Curso de Mestrado do PPG em Ciència do Movimento Humano do CEFD/UFSM "Orientadora, Professora Doutora do Curso de Mestrado do PPG em Ciència do Movimento Humano do CEFD/ UFSM
} 


\section{INTRODUÇÃO}

Antes dos benefícios da modernização industrial, desenvolvimentos da tecnologia, do automóvel, de equipamentos facilitadores do trabalho, da televisão e dos computadores, o estilo de vida ativo era aquele que predominava entre a maioria das pessoas. Porém, com as maravilhas da engenhosidade contemporânea, muitos dos gastos de energia diários foram minimizados. Paralelamente ao declínio do gasto de energia, ocorreu o aumento na ingesta alimentar. Tal situação gerou o potencial para o crescimento alarmante na epidemia de doenças causadas pelo estilo de vida'.

Um estilo de vida saudável implica na contemplação diária de vários aspectos, tais como sono adequado, refeições regulares e saudáveis, abstinência de cigarros, drogas e bebidas alcoólicas e, prática regular de atividade física. Sharkey coloca que pesquisadores do Departamento de Saúde da Califórnia concluíram que os homens poderiam adicionar 11 anos de vida e as mulheres 7 anos, simplesmente seguindo os hábitos do estilo de vida saudável.

Recentemente o Centro de Controle e Prevençäo de Doenças dos Estados Unidos (CDC) relatou que cerca de 250.000 pessoas morrem a cada ano devido ao estilo de vida sedentário. O sedentarismo contribui para $34 \%$ das mortes causadas por doenças cardiacas naquele pais, aproximando-se de U\$ 5,7 bilhōes em custos médicos anuais ${ }^{2}$.

Hábitos tais como atividade física regular e controle de peso fazem parte de um estilo de vida saudável, sendo seus benefícios impressionantes. $\mathrm{Na}$ verdade, a atividade previne inúmeras doenças crônicas, além de auxiliar no tratamento de várias outras, especialmente na prevenção e reabilitação da obesidade

A prática regular de atividade física é essencial à saúde e mais ainda para aqueles que desejam controlar o peso. Dâmaso coloca que em qualquer situação em que a re- dução de peso é necessária, torna-se importante uma mudança nos hábitos e no estilo de vida, sendo a atividade física um recurso essencial à redução e manutenção do peso.

Para controlar o peso, é importante a verificação do balanço energético. "O balanço energético refere-se à ingestão de energia ou às calorias consumidas na dieta, $\ominus$ ao gasto energético às calorias queimadas ao longo das atividades diárias. Se a ingestão excede o gasto, o excesso será armazenado como gordura" . A manutenção do peso corporal, então, implica que a ingestão energética seja, pelo menos, igual ao gasto energético ${ }^{4}$.

Daí a importância de se manter uma prática regular de atividade física para controlar - peso, pois a atividade física é a abordagem mais positiva para este fim ${ }^{1,4,5}$. Nenhuma outra é tão fisiologicamente sadia e positiva. Nem mesmo a dieta parece ser tão eficaz. "Fazer dieta para perder peso é a intervenção de saúde mais sem sucesso em toda a Medicina". Apenas um percentual bem baixo de pessoas (inferior a 10\%) que perderam peso permaneceram em seu peso desejado.

O auxilio da atividade física no controle do peso corporal, segundo Sharkey, devese à conservaçāo ou formação do tecido magro (tecido muscular), que tem a capacidade de queimar calorias, com a concomitante manutenção dos níveis da taxa metabólica basal. Isto garante um consumo energético do organismo aumentado durante o repouso. A atividade física é um dos principais coadjuvantes para a reduçāo do tamanho e volume dos adipócitos e podem proporcionar mudanças significativas quanto a obesidade hipertrófica e hiperplásica ${ }^{3.6}$.

Buscando estudar os efeitos da atividade física sobre a composição corporal e a taxa metabólica basal e diária de adolescentes obesos, Dâmaso ${ }^{4}$ aplicou um programa de atividades físicas, em um periodo de 18 semanas em 27 participantes. A composição corporal dos participantes foi avaliada pré $e$ 
pós intervenção, concluindo que, após o programa, a massa livre de gordura aumentou, diminuiu a adiposidade, bem como aumentou a taxa metabólica basal. Tais resultados expressaram a grande importância da atividade física no controle e tratamento da obesidade. Roa et af realizaram um programa de intervenção multidisciplinar com 30 adolescentes obesos, durante 10 semanas. 0 programa incluiu, dentre outros procedimentos, a prática regular de exercício físico uma hora, três vezes por semana, de forma supervisada, com atividades tipo aeróbica, avaliando-se variáveis antropométricas, cardiovasculares e bioquímicas. De acordo com autores do estudo, a incorporação do treinamento físico foi determinante no descenso do peso corporal, conduzindo à promoção da atividade física no tratamento da obesidade.

É preciso, então, esclarecer a diferença entre atividade física e exercício físico, bem como determinar que tipo de prática é mais recomendada no tratamento da obesidade. Dâmaso ${ }^{4}$ considera "atividade física toda $e$ qualquer açāo que estimule a contração muscular, enquanto o exercicio físico envolve ação resultante de contração muscular planejada e com objetivo específico". A prática de qualquer atividade aumenta o gasto calórico, sendo tal indice relacionado à intensidade e duraçâo. Para contar com esses benefícios è necessário acumular 30 min ou mais de atividade de intensidade moderada ao longo da maioria dos dias da semana. A questão essencial é praticar atividades de forma constante, sejam elas na forma de exercícios planejados em academias ou realizando as atividades do cotidiano. Recomendações atuais colocam que os melhores resultados requerem pouco esforço e precisam apenas das medidas que faziam parte do dia-a-dia das pessoas há algumas décadas ${ }^{5}$.

Cabe mencionar que à medida que a atividade se torna mais intensa, a duraçäo da participaçāo torna-se limitada, motivo pelo qual a atividade moderada foi recomendada para o controle de peso. Para queimar excesso de gordura, deve-se considerar essa intensidade porque a vigorosa não deve ser praticada por um longo tempo, podendo diminuir a gasto calórico. Além disso, a utilização de gordura aumenta com o tempo de prática, com mais gordura sendo queimada após 30 minutos de exercício. "A atividade moderada pode ser continuada por horas sem fadiga excessiva, permitindo significante metabolismo de gordura e gasto calórico" ". Mesmo necessitando de tão pouco tempo de prática e com tantas possibilidades de atividades, a maioria das pessoas não é suficientemente ativa. Em São Paulo, os sedentários correspondem a $46 \%$ da população. Desse índice, um número assustador é de crianças e adolescentes ${ }^{8}$.

O estilo de vida moderno parece ter como característica principal à inatividade física, crescente com o passar dos anos e associada a uma maior mortalidade por doenças crônicas. Principalmente entre os adolescentes, as atuais evidências sugerem que os mesmos estão cada vez mais inativos. Essa evidência parece ser ainda maior entre os adolescentes com excesso de peso. os quais parecem ser menos ativos que seus pares magros. Vários estudos já foram feitos no sentido de verificar o nivel de pratica de atividade física entre os adolescentes. Apesar dos benefícios da prática regular de atividades físicas, não somente para adolescentes obesos, mas para os adolescentes de uma forma geral, observa-se que o sedentarismo entre os jovens é cada vez maior ${ }^{10,11,12}$. Estudos desta natureza vêm mostrando que os adolescentes estão cada vez menos ativos ${ }^{13}$. Este é um fato importante, já que indivíduos ativos quando jovens tendem a ser ativos na vida adulta. Uma vez que os indicios mostram que os adolescentes brasileiros praticam pouca atividade física, há maior probabilidade de permanecerem sedentários, não usufruindo os efeitos benéficos à saúde da atividade física regular.

Nesse sentido, buscou-se, através deste 
estudo, investigar o nível de prática de atividade física dos adolescentes obesos em tratamento no Ambulatório de Obesidade na Infância e Adolescência do Hospital Universitário de Santa Maria, UFSM, RS.

\section{METODOLOGIA}

Este estudo caracteriza-se como uma pesquisa descritiva, uma vez que realizou delineamento da realidade investigada acerca da prática de atividade física em adolescentes obesos. Esta pesquisa foi realizada no contexto do Hospital Universitário de Santa Maria (HUSM), no Ambulatório de Obesidade na Infância e Adolescência, na Universidade Federal de Santa Maria, Santa Maria, RS. Teve como sujeitos 52 adolescentes obesos, sendo o diagnóstico realizado pela equipe médica. Destes 27 são do sexo feminino $(51,92 \%)$ e 25 do sexo masculino $(48,08 \%)$. As idades variaram de 10 e 18 anos, com uma média de 12 anos e 6 meses $(D P=2,14)$, que encontraram-se em tratamento para obesidade no período de abril a outubro de 2002

Para a realização deste estudo fol utilizada uma adaptação do Questionario Internacional de Nivel de Atividade Fisica (IPAQ Versäo 6) de Pardini et al. ${ }^{14}$ para a verificaçăo da prática de atividade física, de acordo com as atividades do dia-a-dia realizadas em uma semana normal, considerando questões sobre prática de atividade física enquanto exercício, na escola, em casa e como meio de transporte.

A coleta dos dados se deu no intervalo da consulta médica, tendo-se o cuidado de anteriormente à aplicaçāo do instrumento, dar explicações necessárias ao entendimento básico do que se tratava, bem como uma anterior exposição da forma como deveriam proceder para respondê-lo. Assim, ficou posto que desejava-se saber quanto tempo o adolescente gastava fazendo atividade física em uma semana normal, bem como a importân- cia de ser sincero nas respostas, mesmo que não se considerasse ativo. $O$ instrumento foi aplicado individualmente, em sala previamente preparada no Ambulatório, ficando o pesquisador à disposição do adolescente para tirar possiveis dúvidas a respeito do instrumento, que por ventura ainda pudessem surgir.

Sua análise foi realizada a partir do critério de Atividade Física Total (AFT). Para determinar a porcentagem dos sujeitos que realizavam atividade física dentro da recomendação atual em saúde, pelo menos 30 minutos por dia, na maior parte dos dias da semana, com somatório semanal de pelo menos 150 minutos por semana de atividade física de intensidade pelo menos moderada. Para atividade física vigorosa o critério foi de pelo menos 60 minutos por semana ${ }^{15}$. Os que não alcançaram esta pontuação foram considerados sedentários.

Os dados foram digitados em microcomputador no programa epi-info versão 2000. Para a análise dos dados foi utilizada a estatística descritiva, média, desvio padrão, freqüências e percentagens de ocorrência, utilizando para isto o Programa Stata Versāo 5.

\section{RESULTADOS E DISCUSSÃO}

A análise dos 52 sujeitos avaliados foi dividida em: a) perfil para os adolescentes obesos avaliados no que tange a sua prática de atividade física e $b$ ) análise das possiveis diferenças entre os sexos para a prática de atividade física dos adolescentes obesos avaliados.

\section{A) Prática de Atividade Física}

A inatividade de um grande número de jovens na atualidade é um fato já observado. Os motivos para isso são inúmeros, e abrangem desde a violência das grandes cidades até o 'comodismo' cada vez mais exagerado 
da vida contemporânea ${ }^{4}$. Diante dessa real circunstância, procurou-se através de uma adaptação do Questionário Internacional de Nível de Atividade Física (IPAQ - Versão 6, de Pardini et al. ${ }^{14}$ ) verificar o número de horas por dia que os sujeitos deste estudo utilizavam assistindo televisăo. A média do grupo para as horas de TV assistidas por dia ficou em 4,65 horas (DP $=1,91$ ), variando de 1 hora por dia (1,92\%) a 8 horas $(9,62 \%)$. 0 tempo de 4 a 6 horas foi o que obteve maior índice de freqüência, perfazendo um total de $51,92 \%$ da amostra. Não houve diferença estatística significativa entre os sexos $(P=$ $0,957)$. A partir dos dados relatados, podese dizer que os adolescentes obesos avaliados por este estudo dedicaram um tempo bastante grande frente à televisão: quase 5 horas diárias. A média de horas frente à televisão encontrada neste estudo foi superior aos valores encontrados na literatura internacional e brasileira.

Estudos como o de Silva \& Malina ${ }^{12}$ verificaram o número de horas frente à televisão em 325 adolescentes de 14 e 15 anos de idade, no municipio de Niterói/RJ, em 32 escolas da rede pública. Eles encontraram uma media de 4 horas diarias frente a televisāo. Dados com amostras do Estado de Sāo Paulo mostraram que a media varia de 3.6 horas diarias ate 3.9 horas diárias em adolescentes de 13 anos . Guedes investigou 313 adolescentes de 15 a 18 anos, em Londrina/ PR. Foi encontrada uma média de 3 a 4 horas diárias de assistência à televisão. Já na literatura internacional, Pate et al. ${ }^{2}$ mostraram uma média de 2 a 3 horas diárias frente à televisão para adolescentes norte-americanos. No Canadá, a média ficou em torno de 2 horas diárias ${ }^{18}$.

Embora muitos estudos já tenham sido realizados acerca do tempo diário de assistência à televisão, seus motivos ainda não estão totalmente esclarecidos. Pode-se apenas inferior que assistir à televisão é um meio de lazer de baixo custo e que oferece a segurança que nem sempre é encontrada nas ruas das cidades maiores. Os ambientes inseguros são, atualmente, uma das barreiras à prática sistemática de atividade física ${ }^{19}$.

O tempo gasto frente a atividades como assistir televisão e computador vem aumentando muito nos últimos anos, coincidentemente ou não com a diminuição da prática de atividade física. Damaso et al. acrescentam ainda que quem sofre com o excesso de peso deve ter cuidado redobrado ao comer diante da televisão, uma prática muito usada atualmente. Cada refeição feita diante da TV aumenta de 38 a 73 minutos o tempo total diário diante do aparelho. Reduzir a freqüência das refeições diante da TV pode auxiliar na redução do tempo de assistencia à mesma e na diminuiçäo da obesidade.

Depois de verificar o tempo de inatividade (nesse caso, horas frente a televisão), $\in$ fundamental verificar o tempo gasto em atividade, seja ele atraves da prática de exercicios e esportes, como de atividades domésticas, escolares ou como meio de transporte, tendo em vista que o importante é adotar um estilo de vida ativo, somando um mínimo de prática ao longo da semana ${ }^{1,4}$. 
Nascimento \& Haeffner, A prática de atividade física em ...

TABELA 1: Distribuição dos adolescentes obesos avaliados a partir da prática e do tempo de prática de atividade física leve, moderada e vigorosa enquanto exercício físico

\begin{tabular}{lcll}
\hline \multicolumn{4}{c}{ Atividade Física enquanto Exercício Físico } \\
\hline & Leve $(\mathrm{N}(\%)$ & Moderada $(\mathrm{N}(\%)$ & Vigorosa $(\mathrm{N}(\%)$ \\
\hline Prática & & $20(28,45)$ & $6(11,54)$ \\
Praticam & $52(100)$ & $32(61.54)$ & $46(88,46)$ \\
Não praticam & & \\
Tempo Prática (Min/Sem) & & $18(34,62)$ & \\
Até 149 min & $2(3,84)$ & $2(3,85)$ \\
Até 59 min & & $4(7,69)$ \\
60 min ou mais & & \\
\hline
\end{tabular}

A prática de atividade física, enquanto exercício físico, encontra-se extremamente reduzida nos adolescentes (Tabela 1). Nesse contexto, nenhum dos adolescentes relatou praticar qualquer exercício de intensidade leve. Dos adolescentes que praticavam algum exercício físico, $38,46 \%$ faziam exercicios moderados e $11,54 \%$ vigorosos. Năo houve diferença estatística significativa entre os sexos em relação a prática dessas atividades (Atividades Moderadas: $P=0,430$; Atividades Vigorosas: $P=0,920$ ). Os dias por semana de pratica de exercícios moderados variaram de 1 a 6 dias, sendo que a freqüência maior ficou entre 2 e 3 dias. Quanto ao exercicio vigoroso, a prática fol ainda menor. Os dias por semana de prática de exercicios vigorosos variaram de 1 a 4 dias, sendo que a freqüência maior ficou em 2 dias. Quanto ao tipo de prática, dos que praticavam atividades moderadas $23,08 \%$ relataram caminhar, $1,92 \%$ participavam de danças gaúchas e $13,46 \%$ o pedalar. Dentre os que praticavam atividades vigorosas, $3,85 \%$ jogavam futebol, $1,92 \%$ vôlei, $1,92 \%$ corrida, $1,92 \%$ judô e $1,92 \%$ dança afro.

O tempo de prática da atividade física leve foi nulo, isto é, nenhum adolescente realizava esta prática. Quanto ao tempo de prática de atividades moderadas, dos 20 adolescentes que relataram prática, $18(34,62 \%)$ praticavam de 30 a 149 minutos por semana, sendo considerados sedentários pelo critério usado. Apenas 02 adolescentes $(3,84 \%)$ praticavam mais de 150 minutos por semana, podendo ser considerados ativos. Em relação ao tempo de prática de atividades vigorosas, dos 6 adolescentes que realizavam atividades vigorosas, $2(3,85 \%)$ praticavam até 59 minutos por semana, sendo considerados sedentários. Outros $4(7,69 \%)$ praticavam 60 minutos ou mais por semana, sendo considerados ativos (Tabela 1).

Buscando verificar a prática de exercicios físicos em adolescentes de São Paulo. Matsudo et al. investigaram 47 adolescentes de 10 a 15 anos. Segundo os pesquisadores, ficou evidente o nāo envolvimento desses adolescentes com exercicios moderados e vigorosos, sendo que o maior número de prática foi de exercícios leves. Silva \& Malina ${ }^{12}$ encontraram resultados semelhantes, em 325 adolescentes de Niterói/RJ, mostrando que a prática de exercicios leves é maior do que a de exercicios moderados e vigorosos, embora a prática em geral tenha sido minima. Tambem Bracco et al. ${ }^{22}$ verificaram em 26 adolescentes ( 7 obesos e 19 não obesos), de 10 e 11 anos, de São Paulo, que os mesmos realizavam mais exercícios leves e pouco tempo foi despendido em exercícios moderados ou vigorosos. Albano ${ }^{10}$ encontrou em 327 adolescentes, de 15 a 19 anos, de uma escola técnica de São Paulo, que não 
ocorrem diferenças significativas na escolha de seus exercícios, sendo os exercícios calmos o campeão de escolha. Em geral, a prática de exercicios encontrada por ele foi bem reduzida entre os adolescentes que participaram do estudo.

Assim, enquanto a literatura coloca que a prática de exercícios leves é mais comum entre os adolescentes, este estudo verificou que a prática de exercícios moderados foi maior. É preciso salientar, ainda, que a prática de exercícios leves foi nula, porém, a prática de exercícios moderados e vigorosos foi mínima, fato que concorda com os achados na literatura' .

Quanto ao tempo de prática (Min/Sem), evidencia-se nas pesquisas encontradas uma média maior do que a encontrada neste estudo, que foi em torno de 90 minutos por semana de prática. Guedes encontrou uma média de 130 minutos por semana de prática de exercícios físicos nos 313 adolescentes investigados em Londrina/PR. Já Fonseca et al. ${ }^{23}$ encontraram, nos 391 adolescentes investigados em Niterói/RJ, uma média de 120 minutos semanais de prática.

Fica evidente que os adolescentes deste estudo praticam exercicios físicos durante um tempo menor do que o encontrado em outras pesquisas. É preciso salientar que os achados não correspondem a amostra de adolescentes obesos. Nesse caso, então, este estudo viria a confirmar as especulações da literatura, as quais colocam que os adolescentes obesos tendem a ser menos ativos do que os não obesos ${ }^{1,3,4}$. A esse respeito, Pasqualini $^{24}$ já afirmava, há 40 anos atrás, que a criança obesa tinha maiores probabilidades de tornar-se um adulto obeso, justamente devido a sua inatividade.

Além da prática de atividade física enquanto exercício físico, o instrumento utilizado também avaliou a prática de atividade física na escola. A Tabela 2 mostra que a prática de atividade física na escola ficou constituída da seguinte forma: dos 50 adolescentes $(96,62 \%)$ que estudam, 35 praticam a Educação Física na escola $(67,30 \%)$. O tempo de prática da Educação Física escolar variou de 30 minutos de aula por semana $(1,92 \%)$ até 135 minutos por semana $(1,92 \%)$. A média de tempo de aula ficou em 60 minutos de Educação Física ao longo da semana $(23,08 \%)$. Quanto ao tipo de atividade prática na aula de Educação Física, nenhum adolescente classificou-a como leve, $46,15 \%$ dos adolescentes classificou-a como moderada e $21,15 \%$ classificou-a como vigorosa. Destaca-se ainda, que outras duas perguntas faziam parte: o tempo gasto na escola andando rápido (Min/dia) e tempo gasto na escola subindo escadas (Min/dia). No entanto, para ambas, nenhum adolescente relatou prática.

TABELA 2: Distribuição dos adolescentes obesos avaliados a partir da prática de atividade física na escola

\begin{tabular}{lcc}
\hline Atividade Física na Escola & $N$ & $\%$ \\
\hline Prática & 17 & 32,69 \\
Não praticam & 35 & 67.30 \\
Praticam & & \\
Tipode Prática & 24 & 46,15 \\
AF Moderada & 11 & 21.15 \\
AF Vigorosa &
\end{tabular}

É importante destacar que os estudos encontrados em relaçāo a prática de atividade física enquanto exercicio físico nāo deixaram claro qual foi o critério de análise para exercicio leve, podendo este nāo corresponder a categoria usada neste estudo, e sim a categoria de atividades domésticas, assistência à televisăo, ou outra. 
A maioria dos adolescentes obesos investigados pratica a Educação Física na escola. Esta é uma prática muito importante, pois além de contribuir ao desenvolvimento biopsicossocial do adolescente, pode também contribuir para uma vida ativa. Guedes \& Guedes ${ }^{25}$ colocam que através da participação nas aulas de Educação Física, crianças e adolescentes podem começar a perceber a atividade física regular como parte normal de suas vidas.

Estudando 127 crianças obesas de Santa Maria/RS, entre 6 e 8 anos de idade, de ambos os sexos, Berleze ${ }^{26}$ verificou que a prática de Educaçāo Fisica escolar era comum a todas, comprovando que as criança obesas estavam engajadas em atividades de vivência motora no contexto escolar. Esse engajamento das crianças em vivências motoras na escola é fundamental para que uma prática de atividade física torne-se duradoura na vida da criança e do adolescente, tornando-se um hábito saudável de vida.

Já Monge ${ }^{27}$, investigando as estruturas interpessoais de adolescentes obesos nas aulas de Educação Física, de ambos os se$x o s$, na faixa etaria de 10 a 12 anos, concluiu que os mesmos eram estigmatizados quanto à participaçāo em atividades físicas. Por esse motivo. preferiam as atividades individuais a coletivas, demonstrando que a Educação Fisica não se constituia em fator importante em seu desenvolvimento social. Vêse que, se por um lado a prática existe, o preconceito contra o obeso em atividades físicas também é uma realidade, fato que pode excluí-los da prática.

Outro dado interessante responde à classificação que os adolescentes obesos deste estudo ofereceram às atividades físicas que compōem a aula de Educaçäo Fisica. A grande maioria classificou sua prática de Educação Física como moderada. Em pesquisa realizada em escolas estaduais de $1^{\circ}$ e $2^{\circ}$ graus de Londrina/PR, Guedes \& Guedes observaram 144 aulas de Educaçāo Física, buscando analisar o tipo de atividades envol- vidas, bem como a intensidade dos esforços físicos realizados durante a mesma. Os autores concluíram que os esforços físicos nas aulas de Educaçāo Física não vêm repercutindo de maneira adequada à saúde, já que o tempo real de aula ficou reduzido a $37 \mathrm{mi}$ nutos no máximo, quase $50 \%$ da aula foi dedicada a atividades de administração e organização, apenas $16 \%$ da aula foi destinada à intensidade moderada, responsável pelas respostas fisiológicas relacionadas à saúde, e somente $6,1 \%$ das aulas eram gastos em atividades aeróbicas. Mechelen \& Kemper ${ }^{13}$ colocam que este é um fato importante, já que indivíduos ativos quando jovens tendem a ser ativos na vida adulta. Assim, a prática da Educaçāo Física na escola pode servir como estímulo a um estilo vida ativo na fase adulta, desde que estimule-os de forma adequada para uma prática voltada à saúde. O professor deve trabalhar em aula, quando houver necessidade, as questões relativas ao preconceito e estigmatização social em relação à obesidade, contribuindo para o nảo afastamento dos adolescentes obesos da prática física, tāo importante para os mesmos.

As atividades domésticas avaliadas, encontram-se na Tabela 3 , onde se observa que a prática de atividades domésticas foi mínima. sendo que a prática de atividades domesticas consideradas vigorosas, tais como carpir, esíregar, arar foi nula. Quanto a prática de atividades domésticas consideradas leves, tais como, tirar pó, lavar louça, arrumar a cama, $55,77 \%$ dos adolescentes relataram praticar e $44,23 \%$ relataram não realizar nenhum tipo de atividade leve em casa. O número de dias de prática variou de 1 a 7 dias por semana, sendo que a freqüência maior ficou entre 1 a 3 dias por semana. Quanto ao tempo gasto neste tipo de atividade $26,91 \%$ dos adolescentes realizam de 10 a 59 minutos por semana e $21,13 \%$ realizam de 60 a 175 minutos por semana. Quanto a prática de atividades domésticas consideradas moderadas, tais como, varrer, rastelar, podar, $88,46 \%$ dos adolescentes relataram não re- 
alizar essa atividade. Apenas $11,54 \%$ relataram realizar atividades moderadas em casa. O número de dias de prática variou de 1 a 6 dias por semana, sendo que a freqüência maior ficou entre 1 e 2 dias por semana. Quanto ao tempo gasto neste tipo de ativi- dade, $9,60 \%$ relataram praticar de 15 a 59 minutos por semana, sendo considerados sedentários pelo critério utilizado. Apenas $1,92 \%$ dos adolescentes relatou realizar mais de 60 minutos por semana, podendo ser considerado ativo.

TABELA 3: Distribuição dos adolescentes obesos avaliados a partir da prática de atividades domésticas leve, moderada e vigorosa

\begin{tabular}{|c|c|c|c|}
\hline \multicolumn{4}{|c|}{ Atividade Física enquanto Atividades Domésticas } \\
\hline & Leve $(\mathrm{N}(\%)$ & Moderada $(\mathrm{N}(\%)$ & Vigorosa $(\mathrm{N}(\%)$ \\
\hline \multicolumn{4}{|l|}{ Prática } \\
\hline Praticam & $29(55,77)$ & $6(11,54)$ & \\
\hline Não praticam & $23(44,23)$ & $46(88,46)$ & $52(100)$ \\
\hline \multicolumn{4}{|c|}{ Tempo Prática (Min/Sem) } \\
\hline Até $59 \mathrm{~min}$ & $18(26,91)$ & & \\
\hline 60 min ou mias & $11(21,13)$ & & \\
\hline Até $149 \mathrm{~min}$ & & $5(9,60)$ & \\
\hline 150 min ou mias & & $1(1,92)$ & \\
\hline
\end{tabular}

Embora a escassez de literatura a respeito da prática de atividades domésticas não permita muitas consideraçōes e comparações, pode-se afirmar que os adolescentes avaliados participam muito pouco dos afazeres domésticos. A participação nas tarefas do lar, principalmente das atividades consideradas moderadas e vigorosas, seria de grande auxilio na manutenção do peso. tendo em vista que o adolescente, envolvido em tal pratica, estaria em gasto energético ${ }^{3}$. A respeito da participaçāo de 127 crianças obesas de Santa Maria/RS, entre 6 e 8 anos de idade, de ambos os sexos, em atividades consideradas domésticas, Berleze verificou que a prática destas atividades era mínima, com um número insignificante de crianças que auxiliavam em casa, nas tarefas do lar. Também Andrade et al. ${ }^{28}$ compararam o padrão de atividade física de adolescentes de 11 a 18 anos de regiões de alto e baixo nível sócio econômico $(n=248)$ do Estado de São Paulo. Eles encontraram diferença estatística significativa entre os grupos na participação das atividades do lar, com a prática de $85,4 \%$ do grupo de baixo nivel e $69,6 \%$ do grupo de alto nível nesse tipo de atividade, demonstrando que a prática de atividade do lar pode sofrer influências do nível sócio econômico, bem como da cultura familiar.

Por fim, resta ainda a verificação da prática de atividades realizadas pelos adolescentes obesos como meio de transporte, para as quais foram coletados dados a respeito do caminhar e do pedalar. A prática destas atividades pode ser visualizada na Tabela 4. Pode-se observar que a prática de uma atividade física como meio de transporte não é muito grande entre os adolescentes obesos avaliados, sendo que o caminhar ainda é mais praticado do que o pedalar. Dentre os adolescentes que praticam a caminhada como meio de transporte, foram encontrados $42,30 \%$, dos quais relataram caminhar para ir e voltar da escola. O número de dias de prática variou entre 4 e 5 dias da semana, sendo a frequêencia maior de 5 dias. Quanto ao tempo de caminhada até a escola, este variou de 10 a 150 minutos, sendo que dos 22 adolescentes que caminhavam como meio de transporte, $34,62 \%$ o faziam até 149 minutos por semana, sendo considerados sedentários pelo critério utilizado. Os outros 
$7,69 \%$ caminhavam 150 minutos por semana, sendo considerados ativos. A diferença entre os sexos quanto ao caminhar como meio de transporte não foi estatisticamente signifi- cativa ( $P=0,148)$. Já quanto a prática do pedalar enquanto meio de transporte, apenas 01 adolescente $(1,92 \%)$ relatou tal prática, realizando-a por 120 minutos por semana.

TABELA 4: Distribuição dos adolescentes obesos a partir da prática de atividade física como meio de transporte

\begin{tabular}{|c|c|c|}
\hline \multicolumn{3}{|c|}{ Atividade Física como Meio de Transporte } \\
\hline & Caminhar $(\mathrm{N}(\%)$ & Pedalar $(\mathrm{N}(\%)$ \\
\hline \multicolumn{3}{|l|}{ Prática } \\
\hline Praticam & $22(42,30)$ & $1(1,92)$ \\
\hline Não praticam & $30(57,69)$ & $51(98,08)$ \\
\hline \multicolumn{3}{|c|}{ Tempo de Prática (Min/Sem) } \\
\hline Até $149 \mathrm{~min}$ & $18(34,62)$ & $1(1,92)$ \\
\hline 150 min ou mais & $4(7.69)$ & \\
\hline
\end{tabular}

Neste estudo, somente a prática da caminhada foi relevante entre os adolescentes obesos investigados enquanto meio de transporte, para ir e voltar da escola. Objetivando a investigação do meio de transporte utilizado por 127 crianças obesas para ir a escola, no Município de Santa Maria/RS, Berleze encontrou um número maior de crianças que iam e voltavam da escola caminhando. No entanto, foi encontrada uma diferença signifjcativa entre as crianças de classes sócio econômicas diferentes. Assim, enquanto as crianças obesas de classe media utilizavam mais o transporte motorizado, as de classe baixa utilizavam mais a caminhada, demonstrando que a prática da caminhada como meio de transporte pode sofrer influências do nivel sócio econômico. Esta influência pode ter ocorrido neste estudo, mas como esta não foi uma variável controlada, năo se pode inferir nada a esse respeito.

A partir da análise de todas as categorias do protocolo, através do critério de Atividade Física Total (AFT) calculou-se a análise somatória de pelo menos 150 minutos por semana de atividade física de intensidade moderada e pelo menos 60 minutos por semana de atividade física vigorosa. ${ }^{19}$. No Gráfico 1. encontra-se o número de adolescentes obesos sedentarios ou ativos, a partir da prática de atividade fisica. Pode-se verificar que dos 52 avaliados, pelos critérios utilizados 33 adolescentes obesos $(63,46 \%)$ são considerados sedentários e $19(36,54 \%)$ podem ser considerados ativos. 
GRÁFICO 1: Distribuiçāo dos adolescentes obesos a partir do nivel de prática de atividade física

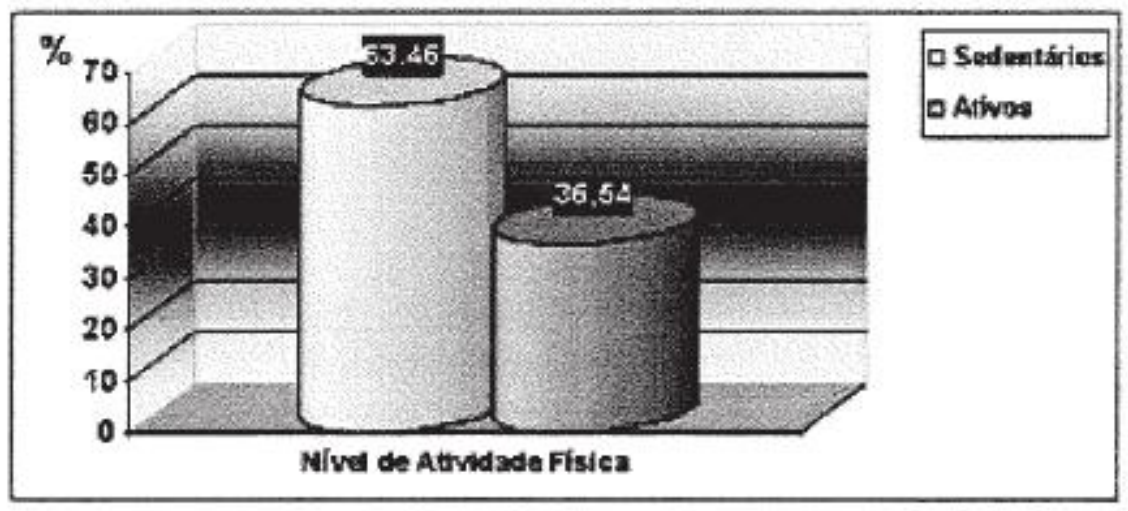

Neste estudo foi encontrado um número muito superior de adolescentes obesos sedentários em relação aos ativos. Este resultado vem ao encontro do que coloca a literatura em relação ao estilo de vida dos adolescentes em geral, bem como dos obesos, sugerindo que este estilo corresponde à inatividade física. Estes achados sugerem que a obesidade infanto-juvenil está cada vez mais associada à inatividade física, para além das questōes nutricionais ou geneticas.

Poucos estudos sobre o nivel de atividade física foram encontrados com amostras de crianças e adolescentes brasileiros

e um menor ainda com amostra composta por obesos . Maitino estudando escolares de 10 a 17 anos de idade da cidade de Bauru, relatou que $42 \%$ dos investigados eram classificados como sedentários. Silva \& Malina ${ }^{12}$ realizaram uma pesquisa no município de Niterói, no RJ, com 325 alunos adolescentes, de 14 e 15 anos de idade, de 32 escolas da rede pública de ensino. Observaram que os adolescentes da amostra praticavam pouca atividade física. Também Matsudo et al. ${ }^{21}$ investigaram o nivel de atividade física de 47 adolescentes de 10 a 15 anos, de ambos os sexos, do litoral norte de São Paulo. Eles encontraram resultados semelhantes ao descrito anteriormente, ou seja, pouca atividade fisica entre os adolescentes escolares que fizeram parte do estudo. Bracco et al. ${ }^{22} \mathrm{com}$ - pararam o nivel de atividade física entre adolescentes de ambos os sexos, obesos e năo obesos, da cidade de São Paulo. A amostra constituiu-se de 26 sujeitos, sendo destes 7 obesos e 19 não obesos. Eles verificaram que os adolescentes não obesos apresentaramse mais ativos do que os obesos. Porém, ambos os grupos apresentaram um perfil insuficiente de atividade física.

$\mathrm{Na}$ literatura internacional, forma encontradas algumas pesquisas realizadas já a algumas décadas com amostras exclusivamente de obesos, todos indicando que ambos os grupos (obesos e não obesos) estavam relativamente inativos, mas os adolescentes obesos eram inativos em um percentual bem maior $^{30.11}$.

B) Prática de Atividade Física e Diferença entre os Sexos

A prática de atividade física dos adolescentes obesos avaliados nāo se mostrou diferente entre os sexos quanto a horas diárias frente à televisão, prática de atividade enquanto exercicio físico, prática de atividade física como meio de transporte e nível de prática de atividade física.

A diferença entre os sexos em relação a prática de Educação Física escolar apresentou-se estatisticamente no limiar de significância, mostrando que os meninos são mais ativos quando comparados com as 
meninas. $\mathrm{Na}$ Tabela 5, observa-se que as meninas praticam menos Educação Física que os meninos, com $44,44 \%$ de meninas que não praticam e apenas $20 \%$ dos meninos. Os achados deste estudo em relaçāo a prática de Educação Física escolar vem ao encontro da literatura em relação ao nível de prática de atividade física. De maneira geral, sabe-se que os meninos são mais ativos que as meninas

TABELA 5: Distribuição dos adolescentes obesos por sexo a partir da freqüência à Educação Física escolar

\begin{tabular}{lll}
\hline Sexo & \multicolumn{2}{c}{ Prática de Educação Física Escolar } \\
\hline \multirow{2}{*}{ Feminino } & Praticam $(\mathrm{N}(\%)$ & Não Praticam $(\mathrm{N}(\%)$ \\
\cline { 2 - 3 } Masculino & $15(55,56)$ & $12(44,44)$ \\
& $20(80)$ & $5(20)$ \\
\hline Total & $35(67,31)$ & $17(32,69)$ \\
\hline$(\mathrm{P}=0,060)$ & &
\end{tabular}

Na Tabela 6, verifica-se a prática de atividades leves e moderadas em casa, para ambos os sexos. Quanto às atividades leves, as meninas praticavam mais que os meninos, apresentando, para a prática um índice de $70.37 \%$ das meninas $\theta 40 \%$ dos meninos. A diferença entre os sexos apresentou-se estatisticamente significativa, denotando que as meninas auxiliam mais nas atividades leves em casa do que os meninos. Já quanto a prá- tica de atividades moderadas em casa, verifica-se que os meninos fazem mais dessas atividades do que as meninas. Assim, 20\% dos meninos realizam atividades moderadas em casa $e$ apenas $3,70 \%$ das meninas. A diferenca estatistica encontra-se no limiar de significâncla, mostrando que os meninos fazem mais atividades moderadas em casa do que as meninas.

TABELA 6: Distribuição dos adolescentes obesos por sexo a partir da prática de atividades domésticas leves e moderadas

\begin{tabular}{|c|c|c|c|c|}
\hline \multirow[t]{3}{*}{ Sexo } & \multicolumn{4}{|c|}{ Prática de Atividades Domésticas } \\
\hline & \multicolumn{2}{|c|}{ Atividade Leve $(\mathrm{N}(\%)$} & \multicolumn{2}{|c|}{ Atividade Moderada $(\mathrm{N}(\%)$} \\
\hline & Praticam & Não Praticam & Praticam & Não praticam \\
\hline Feminino & $19(70.37)$ & $8(29.63)$ & $1(3.70)$ & $26(96,30)$ \\
\hline Masculino & $10(40)$ & $15(60)$ & $5(20)$ & $20(80)$ \\
\hline Total & $29(55,77)$ & $23(44,23)$ & $6(11.54)$ & $46(88,46)$ \\
\hline
\end{tabular}

$(P=0,028) \quad(P=0,066)$ 
As atividades domésticas leves referemse aos trabalhos do lar que não necessitam de muito esforço e que não implicam em um aumento significativo dos batimentos cardiacos e da respiraçăo, tais como tirar pó, lavar louça, cozinhar, estender camas, dobrar e guardar roupas As atividades moderadas referem-se ao varrer, rastelar, podar, etc. A explicação para as diferenças entre os sexos encontradas neste estudo pode ser de ordem cultural. Serrano \& Neto ${ }^{34}$ afimam que cada sujeito nasce no seio de uma família, imbuído de uma cultura específica, significando que a existência do sujeito é condicionada socialmente. "Considerando a complexidade e diversidade de estilos de vida das Sociedades Modernas, pode afirmar-se que as crianças e jovens demonstram nas suas atitudes e comportamentos, as influências do meio social e familiar em que vivem ${ }^{34}$.

Nesse sentido, em nossa cultura, parece ainda existir a divisão das tarefas por sexo, distinguindo-as para homens e mulheres. Assim, as atividades mais realizadas pelas moças corresponderam, neste estudo, às práticas leves, de dentro de casa vistas como 'atividades femininas', enquanto as atividades mais realizadas pelos rapazes referiramse às práticas moderadas, de fora de casa não caracterizadas culturalmente como sendo somente femininas.

Quanto ao nivel de prática de atividade física, ambos os sexos apresentaram-se bastante sedentários e, mesmo sem diferença significativa, pode-se inferir que as meninas são mais sedentárias que os meninos, conforme a Tabela 7 e Gráfico 2.

TABELA 7: Distribuição dos adolescentes obesos por sexo a partir do nível de prática de atividade física

\begin{tabular}{lll}
\hline \multirow{2}{*}{ Sexo } & \multicolumn{2}{c}{ Nível de Prática de Atividade Física } \\
\cline { 2 - 3 } & Sedentários $(\mathrm{N}(\mathrm{S})$ & Ativos $(\mathrm{N}(\%)$ \\
\hline Feminino & $18(66.67)$ & $9(33.33)$ \\
Masculino & $15(60)$ & $10(40)$ \\
\hline Total & $33(63.46)$ & $19(36.54)$ \\
\hline$(\mathrm{P}=0,618)$ & & \\
\hline
\end{tabular}

GRÁFICO 2: Distribuição dos adolescentes obesos por sexo a partir do nível de prática de atividade física

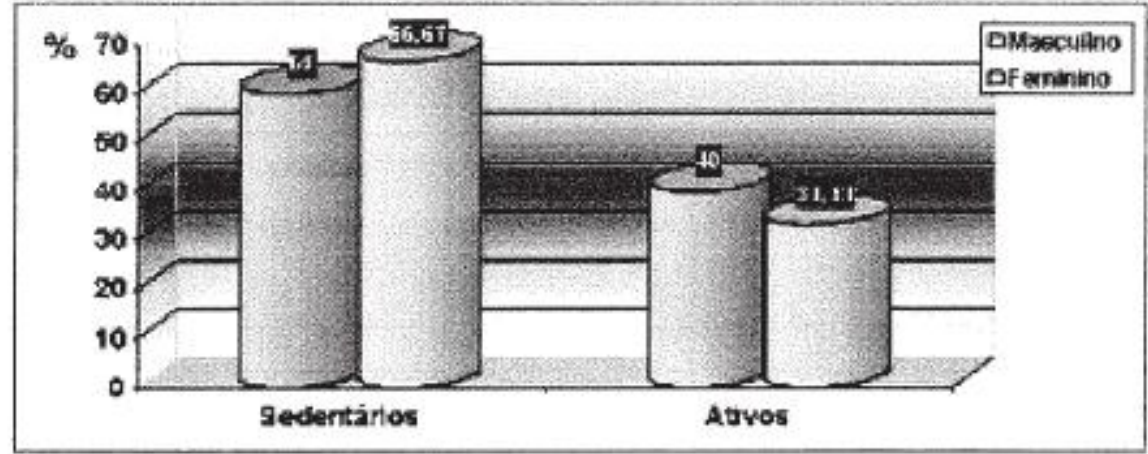


Analisando o nível de atividade física de 325 adolescentes de Niterói/RJ, Silva \& Malina ${ }^{12}$ verificaram que uma maior proporção de meninos foi considerada ativa enquanto eles também apresentaram uma menor proporção de indivíduos muito sedentários. Aproximadamente $85 \%$ dos adolescentes do sexo masculino e $94 \%$ do feminino foram classificados como sedentários. Os meninos, então, mostraram-se mais ativos do que as meninas, com uma diferença de $15 \%$.

Guedes ${ }^{17}$ também verificou o nivel de atividade física por sexo, em uma amostra de 313 adolescentes entre 15 e 18 anos, de Londrina/PR. Os resultados encontrados revelaram que $54 \%$ dos rapazes envolvidos no estudo estavam ativos. Entre as moças, apenas $35 \%$ foram classificadas como tal. A proporção de adolescentes classificados como inativos foi de $65 \%$ entre as moças e $46 \%$ entre os rapazes, comprovando que os rapazes demonstraram ser mais ativos que as moças.

$\mathrm{Na}$ literatura internacional os resultados caminham igualmente, mostrando que meninos sāo mais ativos que meninas. Hagger et at. ${ }^{32}$ analisaram a prática de atividade física em 252 crianças russas e 240 inglesas, de 13 e 14 anos de idade. Os resuitados evidenciaram que os meninos de ambas as nacionalidades foram significativamente mais ativos que as meninas. Na mesma linha de pesquisa, Sallis et al ${ }^{33}$ analisaram 187 crianças de 11 e 12 anos Américo-mexicanas e Angloamericanas. Os autores confirmaram que os meninos foram mais ativos que as meninas.

Parece existir um consenso de que os meninos sejam mais ativos do que as meninas, independente da obesidade, fato que se confirmou neste estudo. A literatura aponta para uma menor atividade por parte do sexo feminino, ainda mais na adolescência, as moças estão sob risco maior de adquirirem hábitos sedentários. No entanto, nenhum estudo em adolescentes obesos comparando o nivel de prática de atividade física por sexo foi encontrado.

\section{CONSIDERAÇÕES FINAIS}

Uma das trocas mais notáveis que vem ocorrendo nas últimas décadas refere-se a tendência à diminuição da prática regular de atividade física, principalmente entre os jovens. Resultados nessa direção foram encontrados neste estudo, podendo-se inferir que o sedentarismo entre os adolescentes obesos é quase uma constante, tendo em vista os altos percentuais encontrados neste estudo.

Contribuindo para tais achados, encontrou-se um tempo de assistência à televisão bastante grande, quase cinco horas diárias. O tempo frente á TV verificado neste estudo é maior do que coloca a literatura, tanto nacional quanto internacional, fato que pode estar contribuindo à inatividade e à obesidade dos adolescentes.

Quanto a prática de exercícios físicos, esta encontra-se muito reduzida entre os adolescentes obesos investigados, sendo dos exercício, o moderado é o mais praticado. Este é um aspecto que pode ser considerado positivo, já que a intensidade moderada é a recomendada para perda e manutenção do peso, bem como para os beneficios gerais a saúde. O tempo de prática destinado a esse tipo de prática, no entanto, encontra-se reduzido e abaixo do que coloca a literatura para adolescentes, fator que pode contribuir ao excesso de peso.

A maioria dos adolescentes pratica as atividades físicas na escola (Educaçäo Fisica), prática esta considera muito importante, não somente para manutenção atual de peso, mas principalmente por estimular para uma vida ativa no futuro. Os meninos participam da Educação Física mais do que as meninas, fato que vem ao encontro do que coloca a literatura em relação a diferença no nivel de atividade física entre os sexos, ou seja, os meninos são mais ativos do que as meninas.

Já em relação a prática de atividades em casa, os adolescentes deste estudo, em geral, participam pouco desses afazeres do- 
mésticos, sendo que esta participação parece estar vinculada a questōes culturais, separando as atividades das meninas das atividades dos meninos. Assim, as meninas participaram mais daquelas atividades considerada culturalmente como sendo "femininas", enquanto os meninos participaram mais de outras atividades, não consideradas culturaimente como sendo exclusivamente femininas.

Quanto as atividades praticadas como meio de transporte, a única praticada pelo grupo investigado que foi significativa referese a caminhada, exclusivamente para ir $e$ voltar da escola. A partir dos achados da lite-

\section{REFERÊNCIAS BIBLIOGRÁFICAS}

\section{SHARKEY, B. Condicionamento físico e} saúde. Porto Alegre: Artmed, $4^{a}$ ed, 1998.

2. PATE, R. et al. Physical activity and public health: A recommendation and the American College of Sports Medicine. JAMA, v. 7, p. 273-402, 1995.

3. FISBERG. M. Obesidade na infância e adolescência. São Paulo: Fundo Editorial BYK, 1995.

4. DÂMASO, A. Nutrição e exercício na prevenção de doenças. Rio de Janeiro: MEDSI, 2001.

5. POLLOCK, M.L. \& WILMORE, J.H. Exercícios na saúde e na doença. Rio de Janeiro: MEDSI, $2^{\mathrm{a}}$ ed, 1993.

6. MACARDLE, W. KATCH, F. \& KATCH, V. Essentials oh exercise physiology. Philadelphia: Lea \& Febiger, 1998.

7. ROA, E.R. et al. Programa de intervenção multidisciplinar em fatores de risco cardiovascular em crianças e adolescentes: obesidade. BIREME/LILACS, 2000. Disponivel em: <www. bireme/opas/oms >.

8. KENSKI, R. Demita seu médico. Super Interessante, 182 ed, nov. 2002, p. 42-50.

9. MAYER, J. \& BULLEN, B. A. Nutrition, weight control and exercise. In: JOHNSON, W. ratura parece que esta pode sofrer influência do nível sócio econômico, porém nada neste sentido pode ser inferido neste estudo.

Finalmente, quanto ao nivel de prática de atividade física, foi encontrado um número muito maior de adolescentes sedentários do que ativos, fato que vem ao encontro da literatura em dois pontos: primeiro, o estilo de vida inativo parece ser cada vez mais o que corresponde à adolescência; segundo, adolescentes obesos tendem a ser mais sedentários que seus pares não obesos, fato que também pode explicar os altos percentuais de inatividade encontrados neste estudo, entre adolescentes obesos.
R. \& BUSKIRK, E. R. Science and medicine of exercise and sport. New York: Harper \& Row, 1974.

10. ALBANO, M.R.C. Comportamento alimentare atividades físicas desenvolvidas por estudantes adolescentes de uma escola técnica de São Paulo. BIREME/LILACS. 2000. Disponivel em: <www. bireme/opas/ oms>

11. ALVES, S.S. et al. Avaliação de atividade física, estado nutricional e condição social em adolescentes. BIREME/LILACS, 2000. Disponivel em: <www. bireme/opas/oms >. 12. SILVA, R. \& MALINA, R.M. Nivel de atividade física em adolescentes do municipio de Niterói RJ, Brasil. Caderno Saúde, v. 16. n. 4, Rio de Janeiro, 2000.

13. MECHELEN, W. \& KEMPER, H.C.G. Habitual physical activity in longitudinal perspective, In: KEMPER, H. The Amsterdam Growth Study. A Longitudinal Analysis of Health, Fitness and Lifestyle. Champaign: Human Kinetics. 1995 (p. 135-158).

14. PARDINI, R. et al. Validação do questionário internacional de nível de atividade física (IPAQ - Versāo 6): estudo piloto em adultos jovens brasileiros. Revista 
Brasileira de Ciência \& Movimento, v. 9, n. 3, p. 45-52, 2001.

15. MATSUDO, S. et al. Questionário internacional de atividade física (IPAQ): estudo de validação e reprodutibilidade no Brasil. Revista Brasileira de Atividade Física e Saúde, v. 6, n. 2, p. 05-18, 2001.

16. ANDRADE, D. et al. Comparison of physical activity involvement in Brazilian teenagers. In: Physical activity, sport and Health. Dallas: 1996.

17. GUEDES, D.P. Prática de atividade física em populações jovens. In: $9^{\circ}$ CONGRESSO DE EDUCAÇÃO FÍSICA E DESPORTO DOS PAISES DE LÍNGUA PORTUGUESA. 2002. São Luís, Anais ..., São Luís: Coleção Prata da Casa, Edição Especial, 2002. p. 6975.

18. KARTZMARYK, P. et al. Television viewing, physical activity and health-related fitness of youth in the family study. Journal of Adolescent Health, n. 23, 1998. P. 318-325. 19. SALLIS, J. F. \& OWEN, N. Physical activity $\&$ behavioral medicine. Thousand Oaks: Sage Publications, 1999.

20. DÂMASO A.R.: TEIXIERA. L. \& CURI, C.M.O.N. Atividades motoras na obesidade. In: FISBERG. M. Obesidade na infância e adolescência. São Paulo: Fundo Editorial BYK, 1995. Cap. 12, p. $91-99$

21. MATSUDO, S.M. et al. Nivel de atividade física em crianças e adolescentes de diferentes regiões de desenvolvimento. Revista da APEF, v. 3, n. 4, 1998.

22. BRACCO, M. et al. Gasto energético entre crianças de escola pública obesas e não obesas. Revista Brasileira de ciência e movimento, v. 10, n. 3, p. 29-35, 2002.

23. FONSECA, V.M.; SICHIERI, R. \& VEIGA, V. Fatores associados à obesidade em adolescentes. Revista da Saúde Pública, v. 32, n. 6, p. 541-549, 1998.

24. PASQUALINI, R. EI libro de los gordos. Buenos Aires: El Ateneo, 1958.

25. GUEDES, D.P. \& GUEDES, J.E.R. Crescimento, composição corporal e desempenho motor de crianças e adolescentes. São Paulo: CLR Balieiro, 1997. 362p.

26. BERLEZE, Adriana. Desenvolvimento motor em crianças obesas: uma análise de contexto. 2002. Dissertação (Mestrado em Ciência do Movimento Humano) Universidade Federal de Santa Maria, Santa Maria, 2002.

27. MONGE, J.C.B. Estruturas interpessoais de escolares obesos nas aulas de educação física. 1999. Dissertação (Mestrado em Ciência do Movimento Humano) - Universidade Federal de Santa Maria, Santa Maria, 1999.

28. ANDRADE, D, et al. Physical activity patterns in female teenagers from different socioeconomic regions. In: Casgrande \& Viviane (ed.) Physical activity and health: physiological, epidemiological and behavioral aspects. Padua: UNIPRESS, 1998. P. 115-122.

29. MAITINO, E.M. Aspectos de risco coronariano em casuística de crianças de escola pública de primeiro grau em Bauru, SP. Revista Brasileira de Atividade Física e Saúde, 17. 2, p. 37-52. 1997.

30. BRUCH. H. Obesity in childhood. IV Energy expenditure of obese children. Am. J. Dis. Child, n. 50, p. 1082-1109, 1940.

31. JOHNSON, M.L. et al. Relative importance of inactivity and overeating in the energy balance of obese high school girls. Am. J. Clin. Nutr, n. 4, p. 37-44, 1956.

32. HAGGER, M. et al. Russian and British children's physical self-perceptions and physical activity participation. Pediatric Exercise Science, v. 10. N. 3, p. 137-152, 1998.

33. SALLIS, J. F. et al. Sex and ethnic differences in children's physical activity: discrepancies between self-report and objective measures. Pediatric Exercise Science, n. 10, p. 277-284, 1998.

34. SERRANO, J. \& NETO, C. As rotinas de vida diária das crianças com idades 
compreendidas entre os 7 e os 10 anos nos meios rural e urbano. In: NETO, C. (Ed.). Jogo
\& Desenvolvimento da criança. Lisboa: Edições FMH, 1997. Cap. 13, ${ }^{\circ}$ 206-225. 